(2) Open Access Full Text Article

\title{
Antipsychotic Treatment Experiences of People with Schizophrenia: Patient Perspectives from an Online Survey
}

\author{
Michael J Doane' \\ Martha Sajatovic ${ }^{2}$ \\ Peter J Weiden $\mathbb{D}^{1}$ \\ Amy K O'Sullivan' \\ Stephen Maher ${ }^{3}$ \\ Jakob B Bjorner (1D ${ }^{3}$ \\ Asia Sikora Kessler ${ }^{3}$ \\ Julia Carpenter-Conlin' \\ Leona Bessonova' \\ Dawn I Velligan (1D) ${ }^{4}$ \\ 'Alkermes, Inc, Waltham, MA, USA; \\ ${ }^{2}$ University Hospitals Cleveland Medical \\ Center, Cleveland, OH, USA; ${ }^{3}$ Optum, \\ Patient Insights, Johnston, RI, USA; ${ }^{4}$ The \\ University of Texas Health Science \\ Center at San Antonio, San Antonio, \\ TX, USA
}

This article was published in the following Dove Press journal: Patient Preference and Adherence

Background: This survey examined the experiences of people living with schizophrenia who have used oral antipsychotics (APs).

Methods: Adults with self-reported physician-diagnosed schizophrenia $(\mathrm{N}=200)$, who were members of an online research participation panel and reported taking one or more oral APs within the last year, completed a cross-sectional online survey that focused on direct report of their experiences regarding APs (eg, symptoms, side effects, adherence). Descriptive analyses were conducted for the total survey sample and for subgroups defined a priori by experience with specific, prevalent side effects.

Results: The mean age of the sample was $41.9(\mathrm{SD}=11.0)$ years, $50 \%$ of participants were female, and $32 \%$ were nonwhite. Overall ratings were positive for medication effectiveness and convenience but negative for side effects. While most participants reported that APs improved schizophrenia symptoms (92\%), 27\% reported APs as having done "more harm than good." Almost all participants (98\%) reported experiencing side effects of APs, with the most common being anxiety $(88 \%)$, feeling drowsy/tired $(86 \%)$, and trouble concentrating $(85 \%)$. Side effects frequently cited as either "extremely" or "very" bothersome were weight gain (56\%), sexual dysfunction (55\%), and trouble concentrating (54\%). Over $80 \%$ reported that side effects had negatively impacted their work and social functioning (eg, social activities or family/romantic relationships). Since initiating treatment, $56 \%$ of respondents had stopped taking APs at some point $(65 \%$ of these due to side effects). Side effects commonly reported as having led to stopping AP treatment were "feeling like a 'zombie"' (22\%), feeling drowsy/tired (21\%), and weight gain $(20 \%)$.

Conclusion: Most participants reported improvements in schizophrenia symptoms associated with the use of APs. However, most participants also reported experiencing numerous bothersome side effects that negatively impacted their work, social functioning, and treatment adherence. Results highlight the unmet need for new APs with favorable benefit-risk profiles.

Keywords: schizophrenia, antipsychotics, side effects, adherence, efficacy, preference

\section{Introduction}

Schizophrenia is a serious mental illness characterized by symptoms including hallucinations, delusions, and thought disorder (ie, positive symptoms), ${ }^{1}$ social withdrawal and apathy (ie, negative symptoms), as well as cognitive difficulties and functional impairment. ${ }^{2}$ Compared to the general population, people with schizophrenia experience lower quality of life, ${ }^{3}$ poorer health, ${ }^{4,5}$ and greater difficulty with day-to-day tasks ${ }^{6}$ and maintaining employment. ${ }^{7}$ These problems are associated with increased economic burden.,
Correspondence: Leona Bessonova Alkermes, Inc, 852 Winter Street, Waltham, MA, USA

Tel + I 78I 6096439

Email Leona.Bessonova@alkermes.com 
Antipsychotic (AP) medications are the primary pharmacologic treatment for people with schizophrenia. ${ }^{10}$ APs are effective in reducing symptoms of schizophrenia, especially positive symptoms. ${ }^{11}$ Second-generation APs (SGAs) may have an improved tolerability profile relative to firstgeneration APs (FGAs; eg, extrapyramidal side effects). While SGAs have sometimes been associated with better adherence than FGAs, ${ }^{12}$ the overall problem of side effect burden is far from resolved. SGA side effects include weight gain, lethargy, and drowsiness. ${ }^{13}$ Suboptimal adherence to APs remains a significant challenge for effective symptom management ${ }^{12}$ and is associated with an increased risk of relapse and rehospitalization. ${ }^{14}$

Patients' subjective experiences with APs are often negative or ambivalent, ${ }^{15}$ and side effects may be a barrier to optimal adherence, creating a cycle of symptom relapse. ${ }^{16}$ Previous work soliciting views of people with schizophrenia regarding treatment with APs indicates that patients are concerned with the impact on their social lives and relationships, with stigma associated with taking these medications, ${ }^{17}$ and with difficulties balancing symptom control and side effects. ${ }^{18}$ Considering that adherence to medication regimens can be influenced by negative beliefs about side effects and their impacts, ${ }^{18,19}$ it is crucial to understand the experience of AP side effects from the patient perspective.

The primary aim of this survey was to gather feedback regarding treatment experiences of individuals with schizophrenia who have taken an oral AP (either FGA or SGA) within the last year. The survey collected information concerning the types, severity, and perceived impact of side effects on participants' overall medication experiences, including impact on social functioning and adherence, and how their past experiences would influence their preferences and willingness to try a hypothetical new AP.

\section{Materials and Methods}

\section{Survey Design}

A cross-sectional, observational online survey was conducted to examine attitudes about AP treatment among those with schizophrenia. Participants were eligible if they were US residents, at least 18 years of age, selfreported a clinician-diagnosis of schizophrenia, received oral APs within a year prior to participating, were under the care of a mental health care professional (MHCP) without hospitalization for at least three months prior to participating, and had not participated in a clinical trial related to psychiatric disorders in the past twelve months (participation in observational studies was not exclusionary). While survey items focused on oral formulations, patients treated with long-acting injectables (LAIs) were not excluded from this study if they had been treated with an oral AP within a year prior to their participation. APs of interest in this study were limited to oral formulations. One aim of this study was to assess the discontinuation of APs, either with or without input from patients' MHCPs; this may differ based on AP formulation. ${ }^{20,21}$ Further, oral APs are frequently prescribed to patients with schizophrenia, ${ }^{22}$ and there are differences in patient characteristics between those receiving oral APs vs LAIs (eg, disease severity, impairment of daily activities ${ }^{23}$ ). This study focused on oral APs to describe patient experiences with these commonly prescribed treatments.

Screening and survey information was self-reported, though participants were identified by Schlesinger Group, a firm specializing in recruiting research participants. Schlesinger Group collaborated with three organizations, each running a pre-existing panel of individuals interested in participating in survey research assembled using inperson, phone, and online recruitment strategies. Initial membership surveys, including demographic, medical, and lifestyle information, were collected from all panel members. The panel administrators employed regular validation procedures to ensure the quality and authenticity of panel members (eg, digital fingerprinting, flagging of suspicious IP addresses, etc). For this survey, Schlesinger Group identified adult panel members with a selfreported mental health diagnosis (based on initial membership surveys) and invited them to participate via email. Participants recruited for this study included those who had experience completing online surveys.

To ensure generalizability, a priori recruitment parameters limited the gender of participants to at most 55\% men, ${ }^{24}$ at least $70 \%$ older than 30 years, and $20 \%$ over the age of 50 . No other a priori parameters were used as quotas for recruitment. Participants completed all materials online via a secure web portal/electronic data capture system used previously in this population. ${ }^{25}$ All participants received an informed consent form, screener, and, if deemed eligible and willing to participate, a survey. The survey was conducted from December 14, 2018 to January 15, 2019. Materials were reviewed and approved by the New England Independent Review Board.

Demographic information (ie, race, ethnicity, age, education, time since diagnosis) was collected via screener and 
survey items. Participants were not asked about specific medications due to concerns over recall and participant burden. The first portion of the survey employed validated instruments, including the Treatment Satisfaction Questionnaire for Medication Version II (TSQMvII), ${ }^{26}$ measuring participant satisfaction with their current AP over the past two to three weeks. The TSQMvII measured participants' satisfaction across four domains: global satisfaction, effectiveness, convenience, and side effects. The response choices for all TSQMvII items use a 7-point Likert-type scale ("extremely dissatisfied" to "extremely satisfied") except for items belonging to the Side Effects domain, which use a 5-point Likert-type scale ("extremely dissatisfied" to "not at all dissatisfied"). Scores for each domain are the average of responses adjusted to range from $0-100$, where higher scores correspond to greater satisfaction.

The remainder of the survey consisted of original items developed by the team to elicit participant feedback regarding their overall experience with APs (see original items in Additional file 1). Content for these items, including symptom and side effect lists, was developed based on several sources, including a focus group study of participants with schizophrenia presented elsewhere, ${ }^{27}$ a review of the literature including existing measures on patient attitudes, and expert input.

Survey items included experience with 12 common AP side effects. The specific side effects were selected on the basis of previous focus group responses and a targeted literature search. The list of 12 side effects consisted of anxiety, gastrointestinal problems, including nausea, dizziness/fainting, dry mouth, feeling a lack of emotion, feeling drowsy or tired, "feeling like a "zombie," involuntary spasms, movements, twitching or stiffness, restlessness, sexual dysfunction, trouble concentrating, and weight gain. Participants were also given an "other" option where they could write in side effects that were not listed. For each side effect experienced, subjective distress was assessed using the term "bothersome" anchored on a 4-point scale (from "not bothersome" to "extremely bothersome"). Other items regarding AP side effects assessed the perceived impact of side effects on social functioning (work, social activities, relationships with family and friends, and romantic relationships), and embarrassment due to experienced side effects.

Participants were also asked whether they had stopped taking their AP with or without agreement from their $\mathrm{MHCP}$, and if medications were stopped, the reason for stopping. MHCP referred to any mental health care professional, not just the MHCP who prescribed a patient's AP treatment regimen. Other items addressed participants' overall assessments of the efficacy and the benefits of their APs.

Finally, participants were asked about their preferences for characteristics of a hypothetical new AP. One item asked participants to directly compare general symptom control versus general side effect experience in a trade-off scenario balancing efficacy and side effects. Other AP preference items asked participants to choose symptoms that they would most like to be controlled, and side effects that they would most want to avoid. For both symptoms and side effects, participants could choose up to 5 from separate lists.

\section{Analyses}

Only completed surveys were accepted (see Additional file 2 - Figure S1). Survey items developed specifically for this study were not combined into composite scales and were scored and analyzed separately. Any AP side effects written in by participants under "other" were included in reporting if written by at least three participants. All analyses were descriptive; no hypothesis testing was performed. Thus, a formal power analysis was not conducted and a sample size of 200 participants was anticipated to allow for reasonable representation of patient characteristics such as age, gender, and race/ethnicity. All analyses were conducted using SAS 9.4 (Cary, NC: SAS Institute Inc.). Categorical data are reported using frequencies and percentages, while continuous data are reported using mean, standard deviation (SD).

Additional cross-tabulations were performed by the extent to which weight gain was reported as bothersome. Weight gain was identified as a stratification variable a priori because it was the only side effect spontaneously reported by a majority of participants in a previous focus group study ${ }^{27}$ as a consequence of AP use. In the previous study, three 90minute focus groups ( $\mathrm{N}=15 ; 5$ participants per group) were conducted with participants with schizophrenia meeting similar eligibility criteria as in the current survey. ${ }^{27}$ The highest levels of weight gain bother ("very" and "extremely bothersome") were combined into a single group due to the conceptual similarity of these responses. Cross tabulations were performed separately for the social functioning and treatment adherence items, which were dichotomized for analyses (presence vs absence of social functioning impact or adherence impact). An additional post hoc analysis 
examined preferences for a hypothetical new AP stratified by duration of illness (less than 10 years vs 10 or more years since diagnosis), as preferences toward APs could change by the duration of time living with schizophrenia.

\section{Results}

\section{Participant Characteristics}

As shown in Table 1, the survey sample of 200 participants with schizophrenia that met eligibility criteria (see

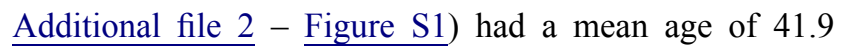
$(\mathrm{SD}=11.0)$ years, was evenly split by gender, and was diverse in terms of race $(32 \%$ identifying as nonCaucasian). Less than half of the sample reported earning a college degree, including an Associate's Degree (15\%), Bachelor's Degree (19\%), or a Graduate Degree (8\%).

Table I Participant Characteristics

\begin{tabular}{|l|l|}
\hline Characteristics & $\mathbf{N}=\mathbf{2 0 0}$ \\
\hline Age, mean (SD) & $41.9(11.0)$ \\
\hline Gender, $\mathbf{n}$ (\%) & \\
Female & $100(50 \%)$ \\
\hline Race, $\mathbf{n}$ (\%) & \\
African-American or Black & $28(14 \%)$ \\
American Indian or Alaskan Native & $3(2 \%)$ \\
Asian & $4(2 \%)$ \\
Caucasian or White & $136(68 \%)$ \\
Muwaiian Native or other Pacific Islander & $0(0 \%)$ \\
Other & $19(10 \%)$ \\
Prefer not to answer this question & $8(4 \%)$ \\
\hline Ethnicity, $\mathbf{n}$ (\%) & $2(1 \%)$ \\
\hline Hispanic/Spanish/Latino origin & \\
\hline Education, $\mathbf{n}$ (\%) & $16(8 \%)$ \\
Less than High School diploma & \\
High School Diploma or GED & $15(8 \%)$ \\
Some College, but no degree & $50(25 \%)$ \\
Associate's Degree or Technical Certificate & $51(26 \%)$ \\
Bachelor's Degree (B.A., B.S., etc) & $30(15 \%)$ \\
Graduate Degree (M.A., M.S., Ph.D. M.D., etc) & $38(19 \%)$ \\
\hline Time Since Diagnosis, $\mathbf{n}$ (\%) & $16(8 \%)$ \\
\hline I year to < 2 years & $27(14 \%)$ \\
3 years to < 3 years & $25(13 \%)$ \\
5 years to < I0 years & $35(18 \%)$ \\
\hline I0 years & $24(12 \%)$ \\
\hline Currently Taking Oral AP, $\mathbf{n}$ (\%) & $89(45 \%)$ \\
\hline
\end{tabular}

Abbreviations: AP, antipsychotic; SD, standard deviation.
Approximately $45 \%$ of participants reported having been diagnosed with schizophrenia for 10 years or longer, and approximately $75 \%$ were diagnosed at least three years prior to the survey.

\section{AP Treatment Experiences Reported Efficacy of APs}

Most participants reported that they were taking an AP at the time of the survey ( $94 \%$, Table 1). Regarding their current AP, participants indicated ratings of global impression of AP medication (mean=62.3, $\mathrm{SD}=25.5)$ and effectiveness of AP medication (mean=61.6, $\mathrm{SD}=25.5$ ) that corresponded to ratings between "somewhat satisfied" and "satisfied" on the TSQMvII. Participant ratings for convenience of AP medication (mean=70.3, $\mathrm{SD}=24.1$ ) corresponded to ratings between "satisfied" and "very satisfied." The mean score on the side effects subscale was lower (mean=51.3, $\mathrm{SD}=25.2$ ), with average responses across the domain corresponding closely to the response option of "somewhat dissatisfied."

Most participants (92\%) reported that their AP improved their schizophrenia symptoms. The largest portion of participants indicated "some improvement" (44\%), followed by "large improvement" (34\%) and "very large improvement" (15\%). Only $2 \%$ (3 participants) indicated that APs worsened their symptoms.

\section{Attitudes Toward APs}

Most of the sample (67\%) acknowledged a need for APs, and $42 \%$ responded "strongly disagree" with the statement "I do not need my oral antipsychotic medications." A total of $27 \%$ of participants agreed with the statement that their APs have done "more harm than good," one-half of participants (51\%) disagreed with this statement, and $23 \%$ were undecided.

\section{Reported Side Effects of APs}

One hundred and ninety-five of 200 participants (98\%) reported experiencing AP side effects. Each of the 12 specified side effects was experienced by at least $70 \%$ of participants. The three most commonly reported side effects were anxiety (88\%), feeling drowsy or tired (86\%), and trouble concentrating $(85 \%)$. Of those reporting experience of respective side effects, weight gain was the side effect with the largest proportion reporting the experience as "very bothersome" (27\%) or "extremely bothersome" (29\%), followed by sexual dysfunction, which was reported as "extremely bothersome" by $33 \%$ of participants and "very bothersome" 


\section{$\llbracket$ Very/ Extremely Bothersome $\quad$ Somewhat Bothersome $\quad$ Not Bothersome}

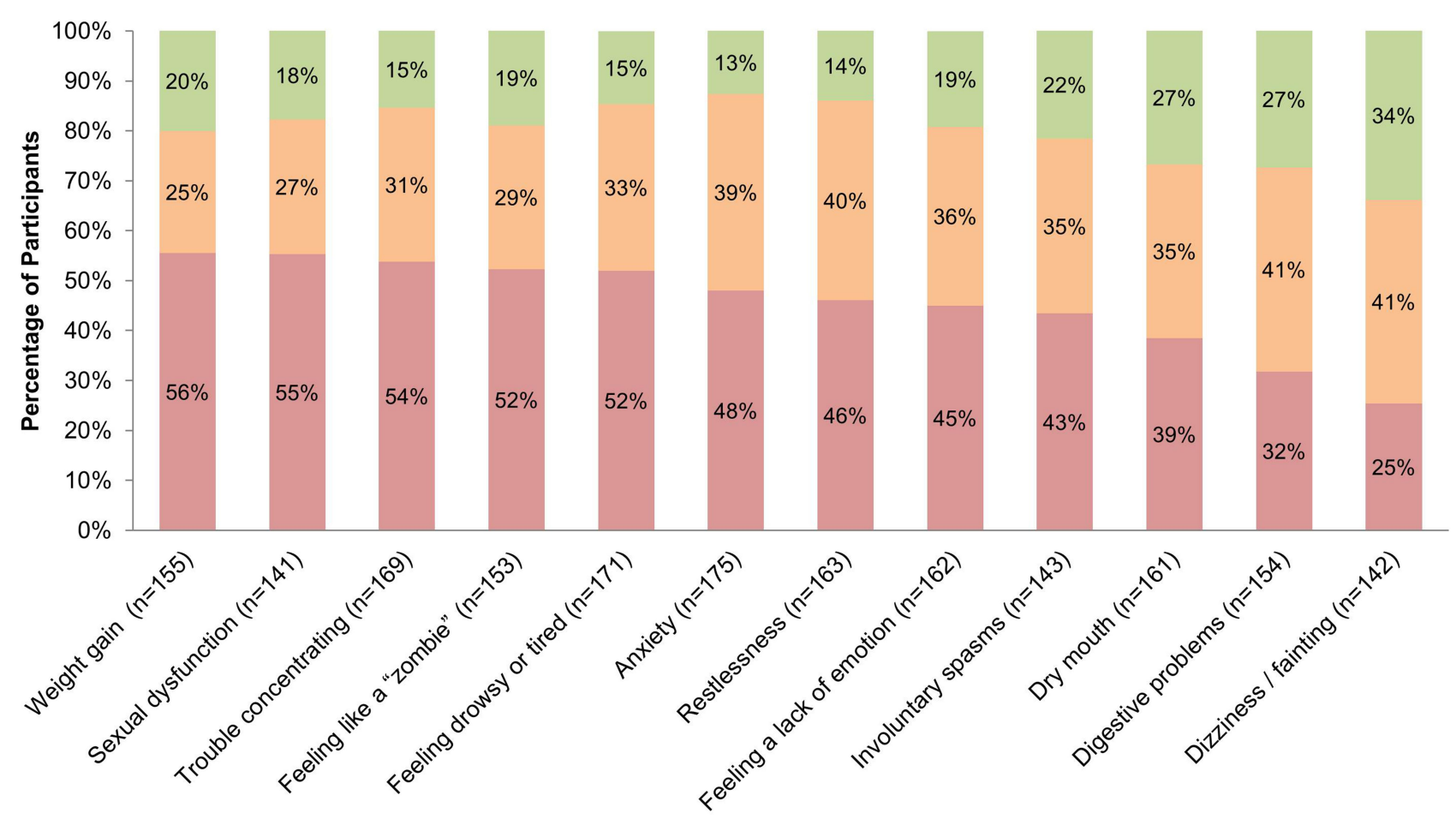

Figure I Bothersomeness of side effects of AP (among participants who experienced side effect).

Notes: The sample size for each side effect only includes participants who reported experiencing the side effect; Side effects are listed from left to right in descending order of percentage of participants who reported "very bothersome" or "extremely bothersome."

by $22 \%$ of those experiencing it (Figure 1 ). The only side effect that was written in by at least three participants was "hunger," reported by three participants (data not shown).

\section{Perceived Impacts of AP Side Effects on Social Functioning}

Most participants (between $82 \%$ and $91 \%$ ) reported some degree of impact of AP side effects across four facets of social functioning queried. For all facets of social functioning, slightly less than half $(42 \%-46 \%)$ of participants reported one of the highest levels of impact ("quite a bit" or "a great deal," Table 2). For example, $45 \%$ of participants indicated that AP side effects have limited their ability to perform paid or unpaid work either "quite a bit" or "a great deal."

Between $33 \%$ and $57 \%$ of participants indicated feeling embarrassment due to AP side effects when considering those experienced. Anxiety was reported as embarrassing by the largest percentage of participants (57\%), followed by sexual dysfunction (53\%), trouble concentrating (51\%), and weight gain $(50 \%)$.

Figure 2 shows the percentage of social impact (including all categories of impact: "very little," "somewhat," "quite a bit," and "a great deal") by the extent to which weight gain was bothersome. For all facets of social functioning, a larger proportion of the group experiencing

Table 2 Perceived Impacts of AP Side Effects on Social Functioning $(\mathrm{N}=195)$

\begin{tabular}{|c|c|c|c|c|c|}
\hline $\begin{array}{l}\text { How Much Have the Side Effects of Your AP } \\
\text { Medications ... }\end{array}$ & $\begin{array}{l}\text { Not at } \\
\text { All }\end{array}$ & $\begin{array}{l}\text { Very } \\
\text { Little }\end{array}$ & Somewhat & $\begin{array}{l}\text { Quite } \\
\text { a Bit }\end{array}$ & $\begin{array}{l}\text { A Great } \\
\text { Deal }\end{array}$ \\
\hline Limited your ability to perform paid or unpaid work? & $14 \%$ & $14 \%$ & $28 \%$ & $22 \%$ & $23 \%$ \\
\hline Limited your ability to engage in social activities? & $9 \%$ & $15 \%$ & $31 \%$ & $21 \%$ & $24 \%$ \\
\hline Interfered with your relationships with friends or family? & $11 \%$ & $21 \%$ & $26 \%$ & $19 \%$ & $23 \%$ \\
\hline Interfered with your romantic relationships? & $19 \%$ & $9 \%$ & $26 \%$ & $19 \%$ & $28 \%$ \\
\hline
\end{tabular}

Note: Percentages are based on 195 participants that indicated experiencing side effects.

Abbreviation: AP, antipsychotic. 


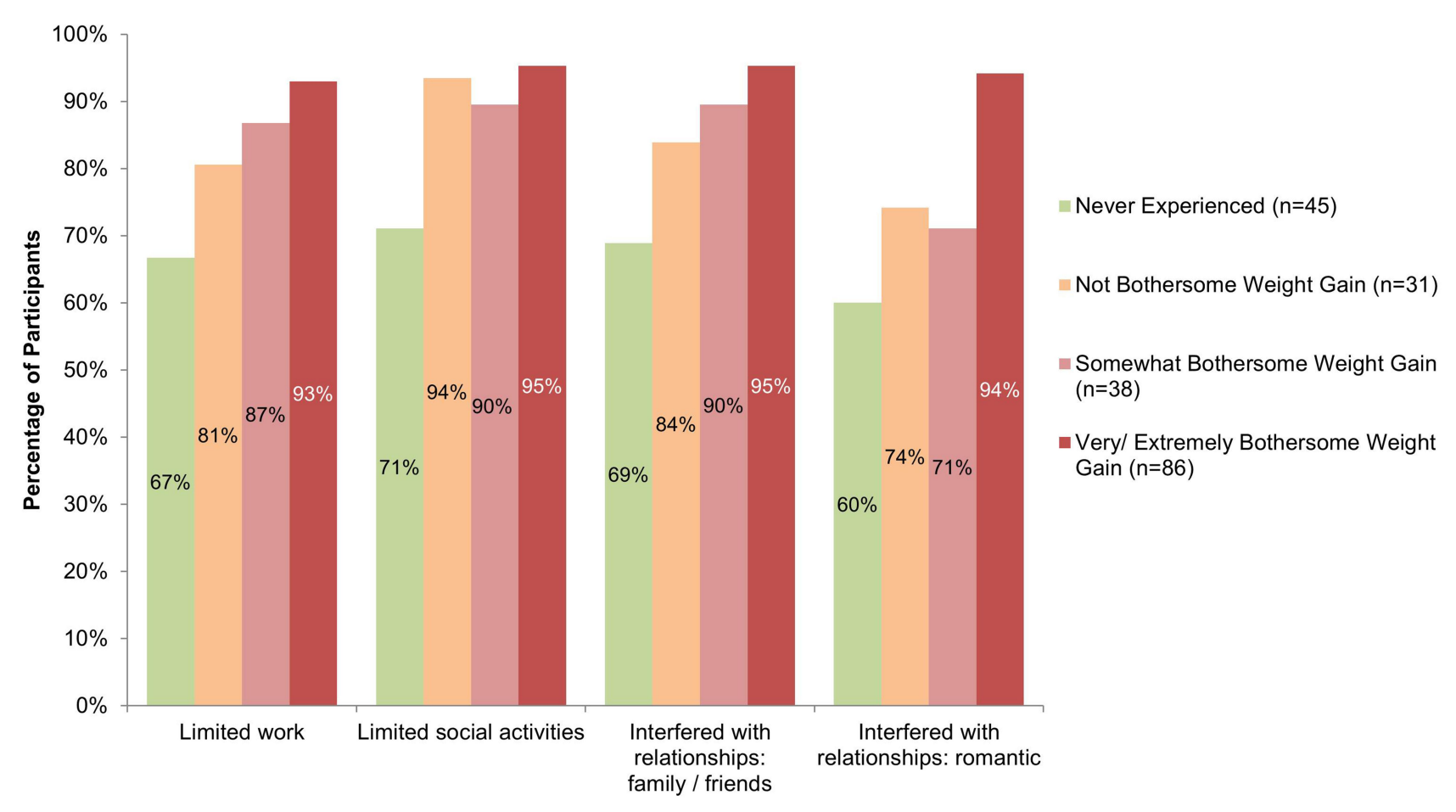

Figure 2 Perceived impacts of AP side effects on social functioning stratified by weight gain experience $(N=200)$.

weight gain reported limitations in social functioning compared to those who did not experience weight gain.

\section{Stopping APs}

More than half of participants (56\%) had stopped taking an AP at some point, either with and/or without agreement of their MHCP. Among all participants, just under one-half (46\%) reported having stopped their AP at some point without the agreement of their MHCP (ie, nonadherence) and close to one-third (31\%) reported having stopped their medication with the agreement of their MHCP at some point. A subset of participants (20\%) had indicated agreement with both options: having stopped their medication with the agreement of their MHCP at some point, and at another time having stopped their medication without the agreement of their MHCP.

The most cited reason for stopping APs was side effects. Among those who reported stopping APs without MHCP agreement, $67 \%$ indicated side effects as a reason, $56 \%$ indicated not liking to take medication as a reason for stopping, and $46 \%$ did not believe the medication was necessary. Among those stopping APs with MHCP agreement, side effects were mentioned by $67 \%$, changing to another medication by $53 \%$, and not liking to take medication by $37 \%$. Among those stopping APs because of side effects (regardless of MHCP agreement), the three most frequently cited side effects were: "feeling like a 'zombie"' $(22 \%)$, feeling drowsy or tired (21\%), and weight gain (20\%).

Figure 3 shows the percentage of participants stopping APs by the extent to which weight gain was bothersome. Among those with "somewhat," "very," or "extremely bothersome" weight gain, approximately half of participants reported having stopped APs without the agreement of their MHCP (51\%-55\%, right part of Figure 3). For those with no weight gain or weight gain that was not bothersome, the percentages are lower (29\%-38\%). In contrast, no clear trend across weight bother groups is seen regarding stopping medication with the agreement of the MHCP.

\section{Preferences for a Hypothetical New AP}

When asked to compare symptoms to be controlled versus side effects to be avoided relative to their current or most recently used AP in a generalized trade-off scenario (Table 3), participants reported a preference for improvement in symptoms rather than reduction in side effects. Most participants (67\%) reported being interested in an AP that would improve symptoms of schizophrenia. However, the highest proportion of participants (45\%) preferred "small improvements in symptoms for no change in side effects" rather than "large improvements in symptoms with slightly worse side effects" (chosen by only $22 \%$ ). One-third of participants (34\%) indicated 


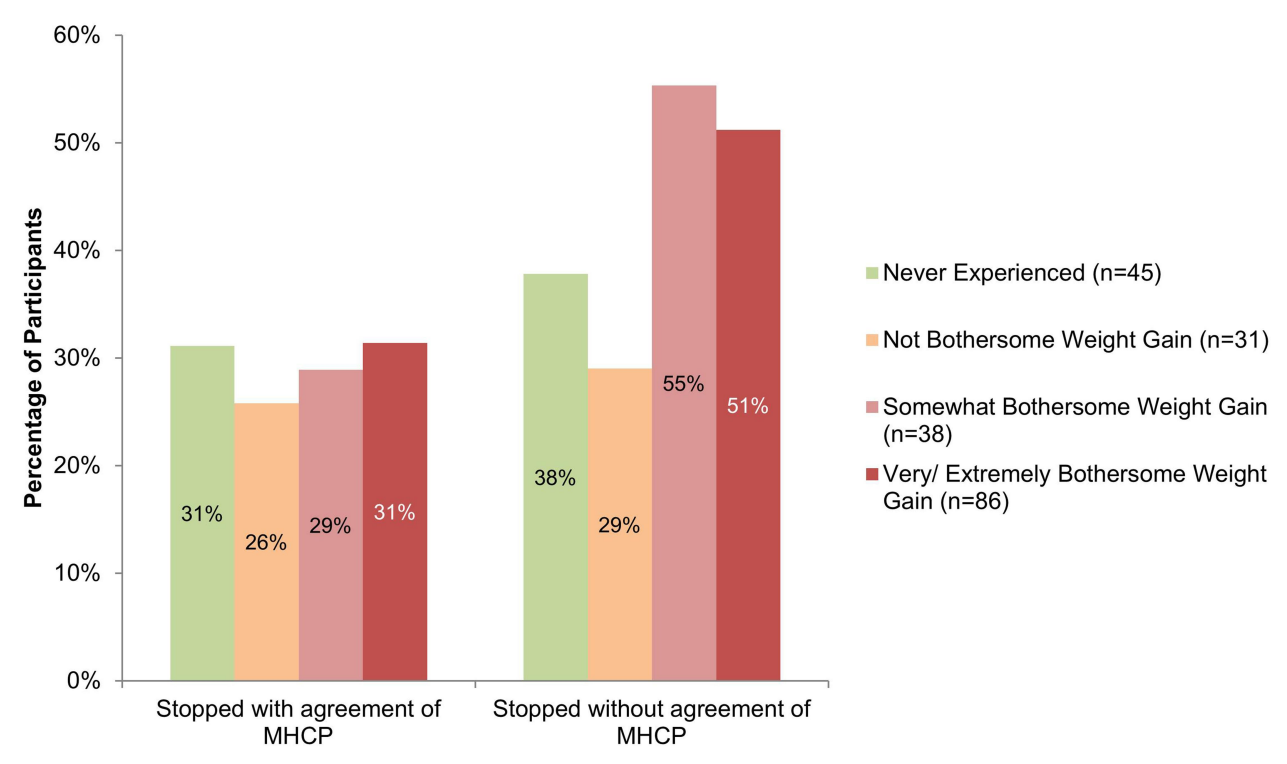

Figure 3 Stopping AP stratified by four categories of weight gain experience $(N=200)$.

Abbreviation: MHCP, mental health care professional.

a preference for medications with improved side effects, with $9 \%$ of all participants indicating a willingness to accept worse symptom control if it meant large improvements in side effects. When stratified by duration of illness, no difference was observed among participants who had been diagnosed for less than 10 years vs 10 years or longer; most participants reported a preference for APs that would improve symptoms of schizophrenia compared with relieving side effects ( $66 \%$ of participants diagnosed for less than 10 years and $68 \%$ of participants diagnosed for 10 years or longer preferred APs that would improve symptoms) (Table 3 ).

In a hypothetical new AP, the symptoms that participants most frequently reported wanting better control for were anxiety (55\%), inability to concentrate or think clearly (45\%), feeling withdrawn from others $(42 \%)$, feeling that others are working together against them (41\%), and auditory hallucinations (40\%). Anxiety was also listed as the most endorsed side effect to be avoided (45\%). Three other common side effects to be avoided were: "feeling like a 'zombie", (39\%), weight gain (38\%), and sexual dysfunction (35\%). (See Additional file 2 - Tables $\underline{\mathrm{S} 1}$ and $\underline{\mathrm{S} 2}$ )

\section{Discussion}

This online survey captured the experiences with APs among 200 participants living with schizophrenia. Most participants had lived with schizophrenia for many years ( $45 \%$ for 10 or more years). The sample was diverse in terms of demographics and educational background. The overall educational level was in line with estimated educational attainment for the US general population. $^{28}$

Table 3 Trade-Offs Between Symptom Relief and Side Effects in an AP (N=200)

\begin{tabular}{|l|l|l|l|l|}
\hline \multicolumn{1}{|c|}{ Compared to Your Current or Most Recent AP, Which of the Following Would You Prefer? } \\
\hline & $\begin{array}{l}\text { Large improvement in } \\
\text { symptoms, but slightly } \\
\text { worse side effects } \\
\text { n (row \%) }\end{array}$ & $\begin{array}{l}\text { Small improvement in } \\
\text { symptoms, but no change } \\
\text { in side effects } \\
\text { n (row \%) }\end{array}$ & $\begin{array}{l}\text { No change in symptoms, } \\
\text { but small improvement in } \\
\text { side effects } \\
\text { n (row \%) }\end{array}$ & $\begin{array}{l}\text { Slightly worse symptoms, } \\
\text { but large improvement in } \\
\text { side effects } \\
\text { n (row \%) }\end{array}$ \\
\hline $\begin{array}{l}\text { Overall } \\
\begin{array}{l}\text { Disease duration } \\
\text { Less than 10 years } \\
\text { I0 or more years }\end{array}\end{array}$ & $\begin{array}{l}90(45 \%) \\
25(28 \%)\end{array}$ & $49(25 \%)$ & $17(9 \%)$ \\
\hline
\end{tabular}

Abbreviation: AP, antipsychotic. 
Participants were mostly satisfied with their current medication except for side effect burden. Almost all participants reported side effects from APs, and each of the twelve side effects listed in the survey was experienced by at least $70 \%$ of participants. The three most commonly experienced side effects were anxiety, feeling drowsy or tired, and trouble concentrating. The most commonly experienced side effects, however, only partially overlapped with those reported as most bothersome, which were weight gain, sexual dysfunction, and trouble concentrating.

Interpretation of participant assessment of side effects is complicated by attribution of schizophrenia symptoms versus treatment side effects. While this report listed symptoms and side effects independently, it may be difficult for participants - and their clinicians - to disentangle these two. We suspect that there may be overlap as the pattern of responses shows that participants endorse the same problem as a symptom and a side effect. For example, akathisia, a movement disorder characterized by a subjective feeling of restlessness and caused by APs, is often misdiagnosed or may be experienced as anxiety. ${ }^{29}$ In our survey and in previously conducted focus groups, ${ }^{27}$ anxiety and trouble concentrating were two frequently reported side effects, but participants also indicated these to be symptoms of schizophrenia. These conditions could be side effects of treatment, ${ }^{30-32}$ but may also be part of the schizophrenia syndrome itself (amotivation, negative symptoms). The ambiguity of attribution of these experiences to syndrome or medication is one of many factors that must be considered to better understand participants' characterization of the side effects associated with APs (also see Kleinman et $\mathrm{al}^{33}$ ).

Almost all participants responded that AP side effects, taken together, had negatively impacted important areas of their lives. This result is in line with previous work. ${ }^{34,35}$ The impacts included work life, social activities, relationships with family and friends, and romantic relationships. Social impacts may in part owe to embarrassment participants indicating experiencing over anxiety, sexual dysfunction, trouble concentrating, and weight gain; these side effects again partially overlapping with those frequently cited as bothersome. Embarrassment over sexual dysfunction may also explain why many more participants in this anonymous survey cited this side effect as compared to the focus groups ${ }^{27}$ ( $71 \%$ vs $26 \%$ ).

Further analyses examined the impact of weight gain, as this was the most prevalent side effect mentioned by participants in focus groups ${ }^{27}$ conducted to inform the current survey. In analyses that stratified the degree to which weight gain was bothersome, a larger proportion of participants with more bothersome weight gain reported a negative impact on their social functioning as compared to those who reported less bothersome or no weight gain. This trend was found across all facets of social functioning, with large differences between the group not experiencing weight gain and the group experiencing the most bothersome weight gain. While causal inferences cannot be drawn from a cross-sectional survey, these differences suggest that weight gain as an AP side effect may affect social functioning in individuals with schizophrenia.

Despite participant reports about the bothersomeness, embarrassment, and social limitations associated with side effects of APs, participants considered side effects in the context of efficacy. Although a clear majority indicated they needed APs to help control symptoms, only half of participants disagreed with a survey item stating that the medications do "more harm than good." These findings, combined with those indicating that almost all participants reported that their AP improved their schizophrenia symptoms, may indicate that most participants recognize the need for their symptom control with APs, even at the cost of experiencing side effects. In addition, a substantial portion of participants valued efficacy over side effect relief, suggestive of an unwillingness to trade symptom control for improvement in side effects. Among participants who chose greater efficacy over side effect relief, most endorsed a small improvement in symptoms for no change in side effects compared to the alternative, a large improvement in symptoms for slightly worse symptoms. Regardless of the duration of illness, most participants reported a preference for APs that would improve symptoms of schizophrenia compared with relieving side effects. This finding is consistent with previous research that has examined treatment preferences among patients living with schizophrenia. For example, Bridges et $\mathrm{al}^{36}$ found that patients with a recent diagnosis of schizophrenia $(<5$ years since diagnosis $)$ cited a reduction in disease symptoms as the most important goal of treatment. The preference for reduced symptoms of schizophrenia has also been demonstrated in samples of patients living with their condition for a longer range of time. ${ }^{37,38}$ The side effects that participants most wanted to avoid in a new hypothetical AP again overlapped with those reported in regards to other facets of experience (frequency, bothersomeness, embarrassment): anxiety, "feeling like a 'zombie,"' and weight gain. These overall participant 
assessments and attitudes about APs are important as previous work has reported that patient attitudes toward APs are closely linked to adherence and quality of life. ${ }^{35,39}$

Considering the personal ${ }^{3-5}$ and societal ${ }^{8,9}$ impacts of schizophrenia, and the association between non-adherence and risk of relapse/re-hospitalization, ${ }^{14}$ adherence is a topic of special importance. In this sample, more than half of participants indicated they had stopped their AP at some point, and nearly half have been non-adherent (stopped without agreement of their MHCP). While it has been reported that negative attitudes regarding AP side effects predict suboptimal adherence, ${ }^{39}$ side effects were the most cited reason for stopping medication in this survey. The side effects most frequently cited as being associated with stopping - "feeling like a 'zombie,"' feeling drowsy or tired, and weight gain-overlapped with those associated with higher degrees of bothersomeness and impact on social functioning. Among those stopping medication without agreement from their MHCP, a greater proportion had experienced weight gain and found it bothersome - a pattern not found among those stopping with the agreement of their MHCPs. While causal links cannot be drawn from these survey data, it is possible that nonadherence may be driven in part by weight gain when considering the impact of weight gain on social functioning, and that participants in this survey indicated that weight gain is one of the most embarrassing side effects of APs.

Several limitations of our survey should be noted. We used a sample of participants living with schizophrenia identified through existing online patient panels. While this ensures that participants had access to, and were comfortable with, the Internet, it may also mean that the sample is not representative of the total population of individuals with schizophrenia. However, age and recruitment parameters were employed to increase the likelihood that the sample would be representative of the larger population of people with schizophrenia. The sample also may not be representative of the larger population in clinical terms, as it necessarily consists of participants who are willing and able to complete an online survey, ${ }^{40}$ which could also mean that our results may have underestimated the burden of schizophrenia. Consistent with this, the sample has a somewhat higher level of education than found in similar samples, ${ }^{41,42}$ perhaps reflecting the fact that this online survey required some familiarity and comfort with web-access and literacy skills to complete the survey items. Systematic bias may also be present due to differences in online competency by age, such that younger rather than older participants may have been more familiar with completing web-based surveys, presenting the possibility of more user error for older participants.

As data were collected through an anonymous selfreported survey, there is no way to verify participants' responses, including diagnosis and treatment history information. Responses could be affected by systematic recall bias. Many of the experiences of symptoms and side effects overlap by participants' descriptions, and so it may be difficult to make dispositive attributions of these experiences to one category or another. With all of these limitations noted, the aim of this study was to gain insight into the subjective experiences of participants, and many of these limitations are intrinsic to that objective. In spite of the limitations, most of which apply to survey research generally, these findings provide useful information that can help clinicians and researchers better understand drivers of suboptimal adherence and factors that may contribute to patient-clinician shared decision-making with respect to medication and behavioral treatments for schizophrenia.

As all analyses are descriptive in nature, no inferences can be made about associations or causality of the variables analyzed.

Cognitive debriefing was not performed for items developed for this survey. However, the content of the survey was based on the prior qualitative focus group study $^{27}$ of participants living with schizophrenia $(\mathrm{N}=15)$, as well as input from representatives from national mental health patient advocacy groups and clinical experts in the fields of schizophrenia and psychometrics, all of whom agreed on the relevance and face validity of the items. Additionally, all phases of design were conducted in accordance with recent reviews of the literature and the FDA regulatory guidance for patient-reported outcomes. ${ }^{43}$

\section{Conclusions}

AP side effects were experienced by most participants and were cited as being associated with limitations on social functioning, difficulties with adhering to a medication regimen, and difficulties in balancing side effect burden with the consequences of not taking APs. When considering frequency, degree of bother, adherence concerns, and desired attributes of APs, participants assessed three side effects as particularly problematic: weight gain, sexual dysfunction, and "feeling like a 'zombie." Participants with more 
bothersome weight gain as a side effect of APs reported social impacts and non-adherence in greater proportions than those experiencing less bothersome or no weight gain.

These results highlight the unmet need for new APs for schizophrenia that maintain or improve effectiveness in controlling symptoms while reducing bothersome side effects.

\section{Abbreviations}

AP, Antipsychotic; FGA, First-generation antipsychotics; MHCP, Mental health care professional; SD, Standard deviation; SGA, Second-generation antipsychotics; TSQMvII, Treatment Satisfaction Questionnaire for Medication Version II; US, United States.

\section{Data Sharing Statement}

The data collected in this study are proprietary to Alkermes, Inc. Alkermes, Inc. is committed to public sharing of data in accordance with applicable regulations and laws.

\section{Ethics Approval and Informed Consent}

All procedures performed in studies involving human participants were in accordance with the ethical standards of the institutional and/or national research committee and with the 1964 Helsinki declaration and its later amendments or comparable ethical standards. The informed consent form, protocol, and survey were approved by the New England Independent Review Board (IRB\# 120180252). All participants provided written consent.

\section{Acknowledgments}

We would like to thank the following mental health advocacy organizations for providing insight for this research: Mental Health America (MHA) and Schizophrenia and Related Disorders Alliance of America (SARDAA). Portions of the research described in this paper were presented as a poster presentation at the 32nd annual Psych Congress, held Oct. 3-6, 2019, in San Diego, California.

\section{Author Contributions}

All authors made substantial contributions to conception and design, acquisition of data, or analysis and interpretation of data; took part in drafting the article or revising it critically for important intellectual content; agreed to submit to the current journal; gave final approval of the version to be published; and agree to be accountable for all aspects of the work.

\section{Funding}

This research was funded by Alkermes, Inc. The study sponsor was involved in research design, data interpretation, and drafting of the article.

\section{Disclosure}

MJD, AKO, and LB are employees of Alkermes, Inc. and own stock in the company. PJW and JCC were employees of Alkermes, Inc. at the time the study was conducted. SM, ASK, and JBB are employees of Optum, Inc., which was paid by Alkermes, Inc. for assistance in designing and conducting this study, performing statistical analysis, and developing this manuscript. MS reports grants from Nuromate, Otsuka, Alkermes, International Society for Bipolar Disorders (ISBD), National Institutes of Health (NIH), Centers for Disease Control and Prevention (CDC), PatientCentered Outcomes Research Institute (PCORI), personal fees from Alkermes, Otsuka, Janssen, Neurocrine, Bracket, Health Analytics, Frontline Medical Communications, publication royalties from Springer Press, Johns Hopkins University Press, Oxford Press, UpToDate, personal fees from American Physician's Institute, MCM Education, CMEology, Potomac Center for Medical Education, Global Medical Education, Creative Educational Concepts, and Psychopharmacology Institute, during the conduct of the study. DIV reports personal fees from Janssen, Otsuka, Alkermes, Lundbeck, during the conduct of the study. The authors report no other conflicts of interest in this work.

\section{References}

1. American Psychiatric Association. Diagnostic and Statistical Manual of Mental Disorders: DSM-5. Washington, DC: American Psychiatric Association; 2013.

2. Hofer A, Baumgartner S, Bodner T, et al. Patient outcomes in schizophrenia II: the impact of cognition. Eur Psychiatry. 2005;20(5-6):395-402. doi:10.1016/j.eurpsy.2005.02.006

3. Medici CR, Vestergaard CH, Hjorth P, et al. Quality of life and clinical characteristics in a nonselected sample of patients with schizophrenia. Int J Soc Psychiatry. 2016;62(1):12-20. doi:10.1177/0020764015585330

4. Kaskie RE, Graziano B, Ferrarelli F. Schizophrenia and sleep disorders: links, risks, and management challenges. Nat Sci Sleep. 2017;9:227-239. doi:10.2147/NSS.S121076

5. Bahorik AL, Satre DD, Kline-Simon AH, Weisner CM, Campbell CI. Serious mental illness and medical comorbidities: findings from an integrated health care system. J Psychosom Res. 2017;100:35-45. doi:10.1016/j.jpsychores.2017.07.004

6. Rempfer MV, Hamera EK, Brown CE, Cromwell RL. The relations between cognition and the independent living skill of shopping in people with schizophrenia. Psychiatry Res. 2003;117(2):103-112. doi:10.1016/s0165-1781(02)00318-9

7. Schennach R, Musil R, Möller H, Riedel M. Functional outcomes in schizophrenia: employment status as a metric of treatment outcome. Curr Psychiatry Rep. 2012;14(3):229-236. doi:10.1007/s11920-0120261-3 
8. Chong HY, Teoh SL, Wu DB, Kotirum S, Chiou C, Chaiyakunapruk N. Global economic burden of schizophrenia: a systematic review. Neuropsychiatr Dis Treat. 2016;12:357-373. doi:10.2147/NDT.S96649

9. Cloutier M, Aigbogun MS, Guerin A, et al. The economic burden of schizophrenia in the United States in 2013. J Clin Psychiatry. 2016;77(6):764-771. doi:10.4088/JCP.15m10278

10. Lehman AF, Lieberman JA, Dixon LB, et al. Practice guideline for the treatment of patients with schizophrenia, second edition. Am J Psychiatry. 2004;161(2 Suppl):1-56.

11. Leucht S, Komossa K, Rummel-Kluge C, et al. A meta-analysis of head-to-head comparisons of second-generation antipsychotics in the treatment of schizophrenia. Am J Psychiatry. 2009;166(2):152-163. doi:10.1176/appi.ajp.2008.08030368

12. Gianfrancesco FD, Rajagopalan K, Sajatovic M, Wang R. Treatment adherence among patients with schizophrenia treated with atypical and typical antipsychotics. Psychiatry Res. 2006;144(2-3):177-189. doi:10.1016/j.psychres.2006.02.006

13. Serretti A, Ronchi DD, Lorenzi C, Berardi D. New antipsychotics and schizophrenia: a review on efficacy and side effects. Curr Med Chem. 2004;11(3):343-358. doi:10.2174/0929867043456043

14. Robinson D, Woerner MG, Alvir JM, et al. Predictors of relapse following response from a first episode of schizophrenia or schizoaffective disorder. Arch Gen Psychiatry. 1999;56(3):241-247. doi:10.1001/archpsyc.56.3.241

15. Sajatovic M, Rosch DS, Sivec HJ, et al. Insight into illness and attitudes toward medications among inpatients with schizophrenia Psychiatr Serv. 2002;53(10):1319-1321. doi:10.1176/appi. ps.53.10.1319

16. Law MR, Soumerai SB, Ross-Degnan D, Adams AS. A longitudinal study of medication nonadherence and hospitalization risk in schizophrenia. J Clin Psychiatry. 2008;69(1):47-53. doi:10.4088/ jcp.v69n0107

17. Kikkert MJ, Schene AH, Koeter MW, et al. Medication adherence in schizophrenia: exploring patients', carers' and professionals' views. Schizophr Bull (Bp). 2006;32(4):786-794. doi:10.1093/schbul/sbl011

18. Lambert M, Conus $\mathrm{P}$, Eide $\mathrm{P}$, et al. Impact of present and past antipsychotic side effects on attitude toward typical antipsychotic treatment and adherence. Eur Psychiatry. 2004;19(7):415-422. doi:10.1016/j.eurpsy.2004.06.031

19. Draine J, Solomon P. Explaining attitudes toward medication compliance among a seriously mentally ill population. J Nerv Ment Dis. 1994;182(1):50-54. doi:10.1097/00005053-199401000-00009

20. Greene M, Yan T, Chang E, Hartry A, Touya M, Broder MS. Medication adherence and discontinuation of long-acting injectable versus oral antipsychotics in patients with schizophrenia or bipolar disorder. $J$ Med Econ. 2018;21(2):127-134. doi:10.1080/ 13696998.2017.1379412

21. Song X, El Khoury AC, Brouillette M, Smith D, Joshi K. Treatment discontinuation of long-acting injectables or oral atypical antipsychotics among Medicaid recipients with schizophrenia. J Med Econ. 2019;22(11):1105-1112. doi:10.1080/13696998.2019.1615927

22. Kaplan G, Casoy J, Zummo J. Impact of long-acting injectable antipsychotics on medication adherence and clinical, functional, and economic outcomes of schizophrenia. Patient Prefer Adherence. 2013;7:1171-1180. doi:10.2147/PPA.S53795

23. Keenan A, Lin D, Shepherd J, et al. Drivers of prescribing long-acting injectable antipsychotics to patients with schizophrenia. Poster presentation at: Psych Congress 2020 Virtual Experience.

24. Aleman A, Kahn RS, Selten J. Sex differences in the risk of schizophrenia: evidence from meta-analysis. Arch Gen Psychiatry. 2003;60 (6):565-571. doi:10.1001/archpsyc.60.6.565

25. McEvoy J, Gandhi SK, Rizio AA, et al. Effect of tardive dyskinesia on quality of life in patients with bipolar disorder, major depressive disorder, and schizophrenia. Qual Life Res. 2019;28(12):3303-3312. doi:10.1007/s11136-019-02269-8
26. Atkinson MJ, Kumar R, Cappelleri JC, Hass SL. Hierarchical construct validity of the treatment satisfaction questionnaire for medication (TSQM version II) among outpatient pharmacy consumers. Value Health. 2005;8(Suppl 1):S9-S24. doi:10.1111/j.15244733.2005.00066.x

27. Bessonova L, Sajatovic M, Saucier C, et al. Treatment experiences of patients with schizophrenia: findings from a qualitative focus group study. Podium presentation at international society for pharmacoeconomics and outcomes research (ISPOR) annual meeting. New Orleans, LA. Value Health. 2019;22(Suppl 2):S33. doi:10.1016/j.jval.2019.04.009

28. United States Census Bureau. Educational attainment in the United States. 2018.

29. Salem H, Nagpal C, Pigott T, Teixeira AL. Revisiting antipsychotic-induced akathisia: current issues and prospective challenges. Curr Neuropharmacol. 2017;15(5):789-798. doi:10.21 74/1570159X14666161208153644

30. Nasrallah HA. Atypical antipsychotic-induced metabolic side effects: insights from receptor-binding profiles. Mol Psychiatry. 2008;13 (1):27-35. doi:10.1038/sj.mp.4002066

31. Bobes J, Garc A-Portilla MP, Rejas J, et al. Frequency of sexual dysfunction and other reproductive side-effects in patients with schizophrenia treated with risperidone, olanzapine, quetiapine, or haloperidol: the results of the EIRE study. $J$ Sex Marital Ther. 2003;29 (2):125-147. doi:10.1080/713847170

32. Ventriglio A, Gentile A, Stella E, Bellomo A. Metabolic issues in patients affected by schizophrenia: clinical characteristics and medical management. Front Neurosci. 2015;9:297. doi:10.3389/fnins.2015.00297

33. Kleinman A, Eisenberg L, Good B. Culture, illness, and care: clinical lessons from anthropologic and cross-cultural research. Ann Intern Med. 1978;88(2):251-258. doi:10.7326/0003-4819-88-2-251

34. Palijan TŽ, Kovačević D, Vlastelica M, Dadić-Hero E, Sarilar M. Quality of life of persons suffering from schizophrenia, psoriasis and physical disabilities. Psychiatr Danub. 2017;29(1):60-65. doi:10.24869/psyd. 2017.60

35. Hofer A, Kemmler G, Eder U, Edlinger $M$, Hummer $M$, Fleischhacker WW. Quality of life in schizophrenia: the impact of psychopathology, attitude toward medication, and side effects. $J$ Clin Psychiatry. 2004;65(7):932-939. doi:10.4088/JCP.v65n0709

36. Bridges JF, Beusterien K, Heres S, et al. Quantifying the treatment goals of people recently diagnosed with schizophrenia using best-worst scaling. Patient Prefer Adherence. 2018;12:63-70. doi:10.2147/PPA.S152870

37. Levitan B, Markowitz M, Mohamed AF, et al. Patients' preferences related to benefits, risks, and formulations of schizophrenia treatment. Psychiatr Serv. 2015;66(7):719-726. doi:10.1176/appi.ps.201400188

38. Rosenheck R, Stroup S, Keefe RSE, et al. Measuring outcome priorities and preferences in people with schizophrenia. Br J Psychiatry. 2005;187(6):529-536. doi:10.1192/bjp.187.6.529

39. Rettenbacher MA, Hofer A, Eder U, et al. Compliance in schizophrenia: psychopathology, side effects, and patients' attitudes toward the illness and medication. J Clin Psychiatry. 2004;65(9):1211-1218. doi: $10.4088 /$ jcp.v65n0908

40. Miller BJ, Stewart A, Schrimsher J, Peeples D, Buckley PF. How connected are people with schizophrenia? Cell phone, computer, email, and social media use. Psychiatry Res. 2015;225(3):458-463. doi:10.1016/j.psychres.2014.11.067

41. Irani F, Seligman S, Kamath V, Kohler C, Gur RC. A meta-analysis of emotion perception and functional outcomes in schizophrenia. Schizophr Res. 2012;137(1-3):203-211. doi:10.1016/j. schres.2012.01.023

42. Lieberman JA, Stroup TS, McEvoy JP, et al. Effectiveness of antipsychotic drugs in patients with chronic schizophrenia. $N$ Engl $J$ Med. 2005;353(12):1209-1223. doi:10.1056/NEJMoa051688

43. US Food and Drug Administration. Guidance for industry: patient-reported outcome measures: use in medical product development to support labeling claims. 2009. 


\section{Publish your work in this journal}

Patient Preference and Adherence is an international, peer-reviewed, open access journal that focusing on the growing importance of patient preference and adherence throughout the therapeutic continuum. Patient satisfaction, acceptability, quality of life, compliance, persistence and their role in developing new therapeutic modalities and compounds to optimize clinical outcomes for existing disease states are major areas of interest for the journal. This journal has been accepted for indexing on PubMed Central. The manuscript management system is completely online and includes a very quick and fair peer-review system, which is all easy to use. Visit http:// www.dovepress.com/testimonials.php to read real quotes from published authors. 